\title{
An fMRI Study of Words Processing in Chinese Language
}

\author{
Pei Xu1, Bing Sun1, Chunqi Chang2, Nan $\mathrm{Hu}^{1 *}$ \\ ${ }^{1}$ School of Electronic and Information Engineering, Soochow University, Suzhou, China \\ ${ }^{2}$ School of Biomedical Engineering, Shenzhen University, Shenzhen, China \\ Email: sdwzpp@163.com, sunbing@suda.edu.cn, cqchang@szu.edu.cn, "hunan@suda.edu.cn
}

Received 24 December 2015; accepted 19 February 2016; published 26 February 2016

\begin{abstract}
Using the blood oxygen levels dependent technology of magnetic resonance imaging (BLOD-fMRI), we aimed to explore the brain activation after visual stimulation by Chinese words. In the current study, 24 healthy volunteers (12 males, 12 females, right-handed, mean age $26 \pm 2$ years) were prospectively included. The event related design was used in the current fMRI study when participants silently read all words appearing in the middle of the screen. Images were processed with Statistical Parametric Mapping 8 (SPM8) software, by using a general linear model (GLM). Group activations were extracted from the 2 nd level group analysis with a threshold of $p<0.001$, and it was shown that the main activated areas by silent reading tasks were regions involved in brain semantic processing, including middle temporal gyrus, fusiform gyrus, supplementary motor area, inferior frontal gyrus, cingulate gyrus, superior parietal lobule and inferior parietal lobule. It was also learnt that superior parietal lobule and middle temporal gyrus are related with semantic understanding, lenticular nucleus are related with semantic processing. This means, in addition to the cerebral cortex, subcortical nuclei is also very important to the processing of words in Chinese language.
\end{abstract}

\section{Keywords}

Functional Magnetic Resonance Imaging (fMRI), Brain Functional Area, Brain Activation

\section{Introduction}

fMRI (functional Magnetic resonance Imaging) is a non-invasive imaging technology which has been prevalently used to study the neural mechanisms of human's brain nowadays. Its basic idea is: When the subjects are affected by experimental stimuluses for evoking visual, auditory or advanced cognitive activities, the flow rate and oxygen saturation level of the blood in the corresponding cerebral cortex areas will change, hence causing local magnetic susceptibility's change which can be detected by MRI systems [1]. fMRI is widely used in the studies of language, memory and cognition systems of the brain [2].

Language study has been greatly concerned, and since the Broca area was discovered in 1861, the study of

${ }^{*}$ Corresponding author. 
neurolinguistic has been one of the most popular areas of the brain research. In the previous studies, neuropsychologists have explored the activation of words processing in the brain. In these studies it was found that a plurality of regions of the brain involved in the processing of the words, with word processing associated with inferior frontal, middle temporal and parietal areas [3] [4]. However, as the volunteers had different backgrounds and different experimental tasks were used in these studies, the activation maps were inconsistent among the experiments, which lead to inconsistent results. In this context, we cannot obtain similar activation maps through similar experiments [5].

In our study, similar participants coming from the north of China, with similar educational background, were selected. Besides, the characteristics of the words which can affect the brain were fully considered, and stimulus groups were consequently matched on word frequency, strokes, radical frequency, the number of shaped nearly words and the level of abstraction. It can be observed that richer and more comprehensive experimental materials were used to observe the brain activation for verifying and complementing the previous study results.

\section{Materials and Methods}

\subsection{Subjects}

Participants (12 males, 12 females) were right-handed, monolingual native speakers of Chinese all of whom had no history of psychiatric or neurological illness. Their ages ranged between 24 to 28 years (mean $26 \pm 2$ years). They were college educated or higher educated, and had normal or corrected-to-normal vision.

\subsection{Stimuli}

Subjects were instructed to attend to and seriously read all stimulus words silently. Event-related fMRI was used in the experiment. The experimental materials were divided into three groups in order to allow the subjects to get adequate rest during the experiment. Concrete nouns, concrete verbs, abstract nouns, abstract verbs, filler words and hash marks randomly presented in the center of the screen, and a central fixation cross was displayed between stimuluses. All experimental materials were of relatively high frequency according to the CMRR corpus [6], mean frequency for words was 30 million, mean strokes for words was 7.94, average number of shape near words was 102.43, mean radical frequency was 91.73 [7].

Subjects kept the states of maintaining a relaxed state, regarding the screen and reading every word silently during the experiment. Following the scan, every participant was required to complete a word recognition test outside the scanner to confirm that participants had been engaged with the task.

\subsection{Functional Magnetic Resonance Imaging}

A Siemens 3T-Trio A Tim with a head coil attached was employed during the data collection. The subjects were fixed to the coil with a soft sponge to reduce head movement. The high resolution anatomical brain MR images were acquired by using a T1-weighed, 3-D gradient-echo sequence. Table 1 was the parameters of the scanning.

\subsection{Data Analysis}

All the Dicom data were converted to NIFTI format by MRIcron, and were subsequently processed using SPM8 (Statistical Parametric Mapping 8). Firstly, images were corrected for slice timing and realigned to the first image using sinc interpolation. Secondly, images were co-registered to the structural T1 images. Thirdly, to match the subjects' structural images to functional images, images were segmented. Gray and white matter images and bias-field corrected structural image were created by SPM8. Fourthly, the images were warped to match the template of the Montreal Neurological Institute (MNI). Finally, the last step of preprocessing was smoothing, and functional images were smoothed by $6 \mathrm{~mm}$ in each direction. After preprocessing, parameters of the images

Table 1. Sequence and parameters of MRI.

\begin{tabular}{ccccc}
\hline Test content & TR $(\mathrm{mm})$ & TE $(\mathrm{mm})$ & Slice thickness $(\mathrm{mm})$ & The field of view (mm) \\
\hline fMRI task & 2000 & 30 & 4 & $240 \times 210$ \\
3-D gradient-echo sequence & 2300 & 2.98 & 1 & $240 \times 256$ \\
\hline
\end{tabular}


were estimated with the general linear model (GLM) to obtain statistical parametric mapping [8]. Single subject and second level statistical contrasts were computed using the typical haemodynamic response function (HRF). The $t$ value of each voxel was obtained for statistical tests, by setting a threshold value $\mathrm{P}<0.001$. After the preprocessing procedure, the activation maps were obtained.

Group analysis was performed according to a random-effects analysis. Activation to each of the experimental word category was compared against the hash mark condition, by setting a threshold value $\mathrm{P}<0.001$, from which activation maps were obtained.

\section{Results}

All subjects completed fMRI experiments according to the instructions, the moving distance of estimated time series of translations was $-2.5 \mathrm{~mm} \sim 2.5 \mathrm{~mm}$, of rotations from $-2^{\circ}$ to $2^{\circ}$. For one of the results of the subjects, activation was observed at middle temporal gyrus, superior temporal gyrus, precentral and central areas, superior parietal lobule and inferior parietal lobule. Table 2 shows the activation of a single subject. The average results from 24 subjects reveal that the activation was located at middle temporal gyrus, superior temporal gyrus, precentral and central areas, cingulate gyrus, posterior cingulate, fusiform gyrus, inferior frontal gyrus, middle frontal gyrus and putamen. Table 3 displays the average activation of the 24 subjects. The corresponding subject brain activations are shown in Figure 1 and Figure 2, respectively.

Table 2. Brain activation and t values of individual subjects when performing tasks.

\begin{tabular}{|c|c|c|c|c|}
\hline Regions activated & BA & Coordinate & Voxels & $\mathrm{t}$ \\
\hline middle frontal gyrus & - & 60045 & 30 & 5.3 \\
\hline superior parietal lobule & 7 & $-27-7251$ & 19 & 5.1 \\
\hline superior temporal gyrus & 38 & $48-270$ & 20 & 4.75 \\
\hline inferior parietal lobule & 40 & $-45-4257$ & 11 & 4.25 \\
\hline middle temporal gyrus & 18 & $-48-54-6$ & 28 & 4.05 \\
\hline middle temporal gyrus & 18 & $-60-60-6$ & 28 & 3.89 \\
\hline superior temporal gyrus & 38 & $-54-393$ & 15 & 3.88 \\
\hline precentral and central areas & 6 & $-54-645$ & 8 & 3.52 \\
\hline
\end{tabular}

Table 3. Average brain activation and t values of 24 subjects when performing tasks.

\begin{tabular}{ccccc}
\hline Regions activated & BA & Coordinate & Voxels & t \\
\hline angular gyrus & 39 & $45-7236$ & 78 & 5.15 \\
middle temporal gyrus & 18 & $51-7227$ & 78 & 5.95 \\
inferior frontal gyrus & 47 & -51366 & 22 & 4.05 \\
cingulate gyrus & 24 & $-3-648$ & 24 & 4.41 \\
medial frontal back & - & 154518 & 6.34 & 4.19 \\
precentral and central areas & 6 & $-51-651$ & 10 & 4.06 \\
putamen & - & $-27-9-3$ & 22 & 3.96 \\
putamen & - & $-27-180$ & 22 & 3.83 \\
middle temporal gyrus & 18 & $-60-57-3$ & 13 & 3.83 \\
fusiform gyrus & 37 & $-42-45-18$ & 1 & 3.69 \\
middle frontal gyrus & 9 & $51-345$ & 1 & 3.67 \\
superior temporal gyrus & 38 & $606-9$ & 2 & 3.62 \\
middle temporal gyrus & 39 & $-51-7215$ & 3 & 3.58 \\
precentral and central areas & 6 & $-24-1554$ & 2 & \\
posterior cingulate & 23 & $6-5712$ & & \\
\hline
\end{tabular}




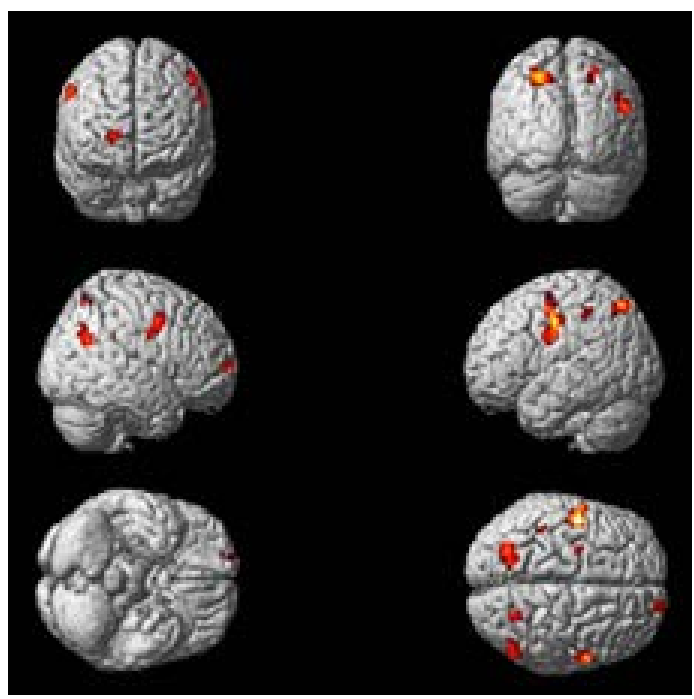

Figure 1. Brain activation of individual subject.

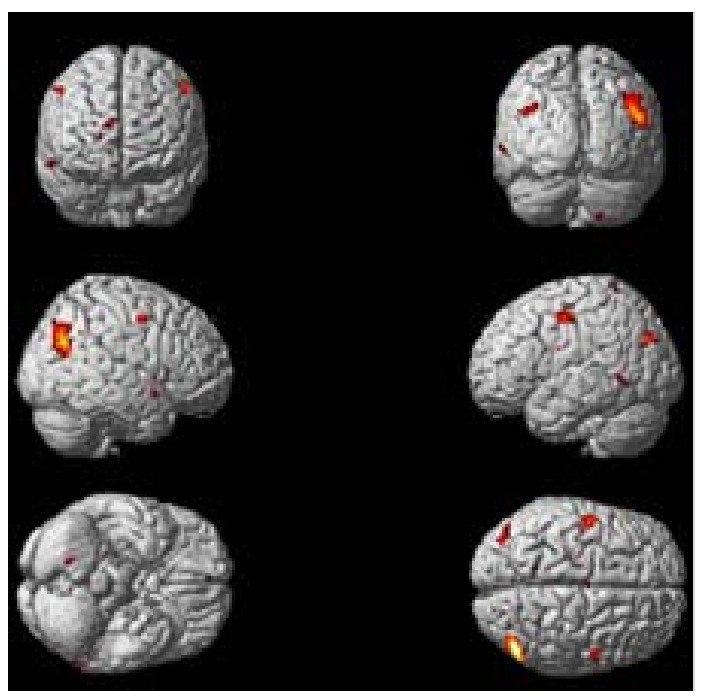

Figure 2. Average brain activation of 24 subjects.

\section{Discussion}

BOLD-fMRI is one of the most important imaging methods in the studies of brain function, in which structural images and functional images were obtained by scanning. Specifically, BOLD signal is decided by increased cerebral metabolic rate of oxygen and a local increase in cerebral blood flow caused by cortical activity [9]. In BOLD-fMRI the BOLD signal is obtained through the changes in the deoxy-hemoglobin content of the blood, and metabolic brain activity is reflected by the signal indirectly, which allows the visualization of neuronal activity changes [10]. In general, BOLD-fMRI has two experimental designs, including block design and eventrelated design, and event-related design was chosen in this paper.

Language areas of the brain have been categorized into movement language center and sensory language center. Movement language center locates at the rear of the inferior frontal gyrus, which is the well-known Broca area. Sensory language center consists of auditory language center and visual language center, where auditory language center is located in the superior temporal gyrus and the rear of middle temporal gyrus and visual language center is located in angular gyrus, i.e. Wernicke area [11] [12]. The task of this experiment was semantic task which to find whether experimental words can cause semantic activations in the brain. To avoid physical disturbance, words frequency, strokes, shape and radical frequency were matched, and hash marks randomly 
appeared during the whole experiment. The results of the experiment showed that activations of the brain were not restricted to a specific area. Activations were shown to be located in both left-hemispheric and right-hemispheric, including middle temporal gyrus (BA39), superior temporal gyrus (BA38), angular gyrus (BA39), inferior frontal gyrus (BA47), middle frontal gyrus (BA9), precentral and central areas (BA6), superior parietal lobule (BA7) and inferior parietal lobule (BA40). Through the results of this experiment, the activation areas included the classic language regions and cortical regions, which is consistent with the results of Tan [13]. Our results indicated that superior parietal lobule and angular gyrus are related to semantic understanding. Besides, the activation of putamen was related to semantic processing of Chinese words, and this activation may be due to the information transformation in corpus callosum. The results indicated that in addition to the cerebral cortex, the nucleus under the cortex is also of great importance to language processing [14]. Compared with the key press reaction task of Tan's study, the task of this study only needs silent reading which can greatly reduce head movements of the subjects, hence reducing artifacts. On the other hand, compared with silent reading, the key press reaction task can increase the degree of participation of subjects. To make up for this shortage, in this study, subjects were asked to fill in the test volume after scan, and the data of the subjects whose correct rates are more than $60 \%$ are selected. Compared with the experimental materials of Pulvermüller's study [15], the experimental materials of this study matched sufficient physical characteristics to avoid physical disturbance, so a more stable result can be obtained.

\section{Conclusion}

In our study, a well designed experiment has been performed to study the words processing mechanism of brain for Chinese language, where words frequency, strokes, shape and radical frequency have been carefully matched. The experiment results revealed that superior parietal lobule as well as middle temporal gyrus and lenticular nucleus were related to semantic understanding and semantic processing, respectively. This implied that, in addition to the cerebral cortex, subcortical nucleus was very important to the processing of Chinese language. The deficiency of this study is that when the subjects are participating in the trials, reading words silently may not guarantee the participation of the subjects. Therefore, reading words silently and key press reaction task can be combined to design experiments in future works.

\section{Acknowledgements}

This work was supported by the Natural Science Foundation of the Higher Education Institutions of Jiangsu Province (14KJB310020), the Special Fund of Industrial Technology Innovation of Suzhou (SYS201521), and 973 Project of Science and Technology (2012CB720702).

\section{References}

[1] Liu, C. (2013) Susceptibility-Weighted Imaging Using in Brain Tumor. Chinese Journal of Medical Imaging, 23, 265-272.

[2] Yang, G., Zhang, Q., Zhang, Y., Shen, J., Zhang, X. and Chen, Q. (2009) An FMRI Study for Different Cognition Components of Working Memory in Schizophrenia Patients. Journal of China Medical Imaging, 20, 758-761.

[3] Kemmerer, D., Rudrauf, D., Manzel, K. and Tranel, D. (2012) Behavioral Patterns and Lesion Sites Associated with Impaired Processing of Lexical and Conceptual Knowledge of Actions. Cortex, 48, 826-848. http://dx.doi.org/10.1016/j.cortex.2010.11.001

[4] Vigliocco, G., Vinson, D.P., Druks, J., Barber, H. and Cappa, S.F. (2011) Nouns and Verbs in the Brain: A Review of Behavioural, Electrophysiological, Neuropsychological and Imaging Studies. Neuroscience and Biobehavioral, 35, 407-426. http://dx.doi.org/10.1016/j.neubiorev.2010.04.007

[5] Zhang, L. Jin, Z., Li, K. and Liu, G. (2013) Cerebral Activations during Verb Generating and Picture Naming: A Study Using Functional MR Imaging. Radiol Practice, 28, 251-255.

[6] Ping, L., Zhen, J. and Li, H. (2004) Neural Representations of Nouns and Verbs in Chinese: An fMRI Study. NeuroImage, 21, 1533-1541. http://dx.doi.org/10.1016/j.neuroimage.2003.10.044

[7] Moseley, R.L. and Pulvermüller, F. (2014) Nouns, Verbs, Objects, Actions, and Abstractions: Local FMRI Activity Indexes Semantics, Not Lexical Categories. Brain and Language, 132, 28-42. http://dx.doi.org/10.1016/j.bandl.2014.03.001

[8] Wang, J. (2011) The Asymptotic Theory of Generalized Linear Models. M.A. Thesis, Guangxi University, Guangxi. 
[9] Zhuang, Y., Shen, J., Xu, J., Zhou, Y. and Lu, Q. (2007) BOLD-FMRI on Aphasia in Patients with Ischemic Stroke: A Pilot Study. Chinese Journal of Medical Imaging, 17, 1143-1145.

[10] Zhang, H., Yan, Z. and Lin, B. (2013) Study of Visual Cortex of Children Amblyopia with Blood Oxygen Level Dependent-Functional Magnetic Resonance Imaging. International Eye Science, 13, 918-920.

[11] Ma, L., Tang, Y., Wang, Y., et al. (2002) Mapping Cortical Areas Associated with Chinese Word Processing with Functional Magnetic Resonance Imaging. Chinese Journal of Radology, 36, 198-201.

[12] Chen, S., Zhang, Y., Zhang, X. and Xiao, Z. (2007) Specific Neural Basis of Chinese Idioms Processing: An EventRelated Functional MRI Study. Chinese Journal of Radology, 41, 930-933.

[13] Tan, L., et al. (2003) Neural Systems of Second Languages Reading Are Shaped by Native Language. Human Brain Mapping, 18, 158-166. http://dx.doi.org/10.1002/hbm.10089

[14] Jiang, M. (2011) FMRI Variability and the Localization of Languages in the Xinjiang Uygurs Brain. Ph.D. Thisis, Xinjiang Medical University, Xinjiang.

[15] Pulvermüller, F., Lutzenberger, W. and Preissl, H. (1999) Nouns and Verbs in the Intact Brain: Evidence from EventRelated Potentials and High-Frequency Cortial Responses. Cerebral Cortex, 9, 497-506.

http://dx.doi.org/10.1093/cercor/9.5.497 